ACCEPTED MANUSCRIPT

\title{
Prediction of a metallic phase for Cs3Pentacene compound
}

To cite this article before publication: Albert Guijarro et al 2018 Mater. Res. Express in press https://doi.org/10.1088/2053-1591/aacc51

\section{Manuscript version: Accepted Manuscript}

Accepted Manuscript is "the version of the article accepted for publication including all changes made as a result of the peer review process, and which may also include the addition to the article by IOP Publishing of a header, an article ID, a cover sheet and/or an 'Accepted Manuscript' watermark, but excluding any other editing, typesetting or other changes made by IOP Publishing and/or its licensors"

This Accepted Manuscript is $@ 2018$ IOP Publishing Ltd.

During the embargo period (the 12 month period from the publication of the Version of Record of this article), the Accepted Manuscript is fully protected by copyright and cannot be reused or reposted elsewhere.

As the Version of Record of this article is going to be / has been published on a subscription basis, this Accepted Manuscript is available for reuse under a CC BY-NC-ND 3.0 licence after the 12 month embargo period.

After the embargo period, everyone is permitted to use copy and redistribute this article for non-commercial purposes only, provided that they adhere to all the terms of the licence https://creativecommons.org/licences/by-nc-nd/3.0

Although reasonable endeavours have been taken to obtain all necessary permissions from third parties to include their copyrighted content within this article, their full citation and copyright line may not be present in this Accepted Manuscript version. Before using any content from this article, please refer to the Version of Record on IOPscience once published for full citation and copyright details, as permissions will likely be required. All third party content is fully copyright protected, unless specifically stated otherwise in the figure caption in the Version of Record.

View the article online for updates and enhancements. 


\title{
Prediction of a metallic phase for $\mathrm{Cs}_{3}$ Pentacene compound
}

\author{
A. Guijarro \\ Departamento de Química Orgánica and Instituto Universitario de Síntesis Orgánica, \\ Universidad de Alicante, San Vicente del Raspeig, 03690 Alicante, Spain. \\ J.A. Vergés \\ Departamento de Teoría y Simulación de Materiales, Instituto de Ciencia \\ de Materiales de Madrid (CSIC), Cantoblanco, 28049 Madrid, Spain.*
}

(Dated: June 6, 2018)

\begin{abstract}
New possible structures for the compounds formed by intercalation of an alkali metal into a molecular crystal formed by polycyclic aromatic hydrocarbon $(\mathrm{PAH})$ molecules have been theoretically searched for. Among them, a crystalline framework in which PAH molecules show a slipped parallel geometry provides an interesting alternative to the usual herringbone arrangement shown by pristine crystal structures of PAHs. While these different crystalline structures are energetically unfavorable for potassium alloying, they are energetically preferred at the highest cesium load (3:1 stoichiometry). The most remarkable feature of the proposed structure is the avoidance of the magnetic instability that leads to insulating phases of herringbone crystals when a 3:1 proportion of metal-Picene is reached. After the evaluation of the corresponding energies of formation, a robust metallic scenario is found for $\mathrm{Cs}_{3}$ Pentacene, making it possible the observation of superconductivity.
\end{abstract}

PACS numbers: 71.28.+d, 36.40.Cg, 71.10.Fd, 71.27.+a

\section{INTRODUCTION}

Intercalation of molecular crystals formed by certain polycyclic aromatic hydrocarbon (PAH) molecules like phenanthrene or picene with alkali or alkaline earth metals (occasionally also with lanthanum and samarium) has originated a new class of superconducting materials of very promising characteristics. The pioneering work of Mitsuhashi et al. ${ }^{1}$ showed superconductivity of potassium-intercalated picene at $7 \mathrm{~K}$ or $18 \mathrm{~K}$ depending on sample processing. Later, other PAH crystals showing a similar laminar structure in which planes are formed by elongated molecules stacking in a herringbone structure have also shown superconducting properties according to the temperature behavior of magnetic susceptibility. The review by Kubozono et al. provides a good starting point to learn about this emerging field ${ }^{2}$. Additional revision work can be found in Refs.(3) and (4). The shielding fraction of the material showing superconductivity properties has always been very small, for example, $1.2 \%$ for one of the $\mathrm{K}_{3}$ Picene samples analyzed in 2010 (Ref.(1)). This fact suggests that superconductivity occurs within a mixture of crystalline phases where the more abundant ones are also the least interesting. This plausible interpretation requires a new rethinking of the theoretical aims in the modeling of these compounds. Minor phases endowed with a robust metallic character are likely to be quite relevant to the explanation of the observed superconductivity. This in turn means that the vast majority of the reported theoretical work, always driven by energetic criteria, has pointed towards intercalated structures that may have only a marginal relation with superconductivity. The possible existence of unknown phases even if they are not at the bottom of the well of thermodynamic stability has been the main motivation of our quest.

Usually, large PAH molecules crystallize in phases showing a stacking of layers in which the dominant figure is a herringbone arrangement favoring $\mathrm{CH}-\pi$ binding among organic

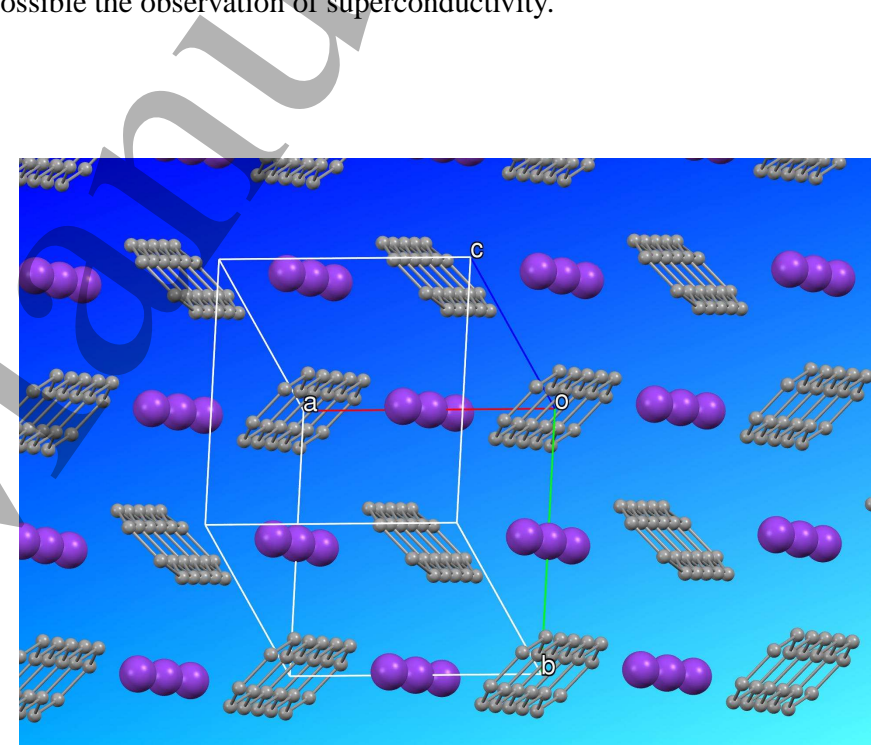

FIG. 1. (Color online) Picture of the 3:1 cesium pentacene compound showing a herringbone arrangement of the organic molecules that is not very different from the one existing in the pristine phase. Only a two-dimensional layer is shown by the figure. Next layers hold the same structure but are somewhat shifted in order to gain density.

molecules ${ }^{5}$. Apparently, this kind of molecular arrangement is preserved after the reaction with metals. Experimental structural evidence is scarce ${ }^{6,7}$. Figure 1 provides a picture of this structure for a 3:1 metal-pentacene compound. Recently, the possible existence of quite different phases has been considered both experimentally ${ }^{8}$ and computationally ${ }^{9}$. This new phase shows a space group change from triclinic to monoclinic in both cases although important differences between the experimentally measured sample and the theoretical prediction exist. The experimental fitting of the X-ray diffraction pattern of this new phase was done keeping a herringbone structure, while in the numerical study, depending on the specific initial seed, the same arrangement didn't hold and spontaneously evolved to a slipped parallel geometry. Figure 


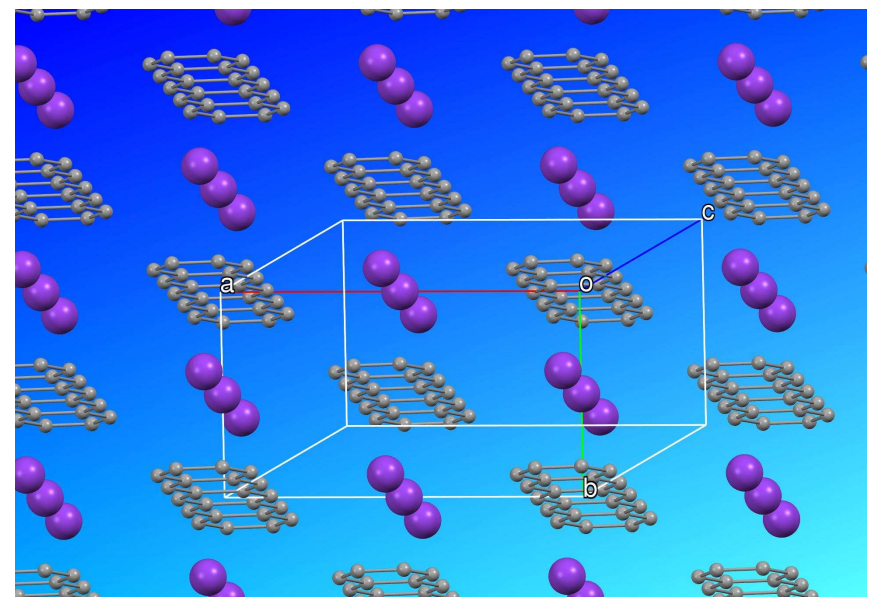

FIG. 2. (Color online) Picture of the 3:1 cesium pentacene compound showing the alternative slipped parallel arrangement of the organic molecules. The figure shows just a two-dimensional layer.

2 provides an image of the proposed structure for a 3:1 metalpentacene compound. In spite of these structural differences, both experiment and theory predict a metallic scenario at low temperatures that makes possible the occurrence of superconductivity. Unfortunately, the new phase of $\mathrm{K}_{3}$ Pentacene is rather unfavorable from an energetic point of view⿳9. Only a high temperature synthesis might yield significant amounts of this phase within a polycrystalline heterogeneous sample. Since intercalation with potassium occurs already at low temperatures (e.g. room temperature), it is not surprising that standard synthetic protocols based on a gradual heating of the reagents fail to provide enriched enough samples with the corresponding superconducting signatures.

In this paper, we analyze the energetics of cesium compounds showing that the alternative crystalline phase not only is metallic for a 3:1 Cs Pentacene stoichiometry but it is also as stable as the usual phase based on a herringbone pattern. This is a specific property of the $\mathrm{Cs}_{3}$ Pentacene compound that is not shared by $\mathrm{Cs}_{3}$ Picene, for example. When the alloying of cesium and picene forming slipped parallel structures is considered, our results show good energetic stabilization of these structures but always smaller than that shown by the extensively studied herringbone compounds. Perhaps, the most important feature shown by the proposed slipped parallel structures is that bandwidths of Lowest Unoccupied Molecular Orbital (LUMO) and LUMO+1 bands are larger than in the herringbone structure. In this way, a metal-insulator transition at low temperature is avoided.

The rest of the paper is organized as follows. Section II is devoted to give some details of the methods and procedures used in this work. Section III presents our main computational results together with some discussion of them. The work ends with a few final concluding remarks (Section IV).



FIG. 3. (Color online) Density of states (DOS) changes due to intralayer cesium doping of pentacene in the herringbone structure. From top to bottom, (a) pristine pentacene, (b) 1:1 cesium pentacene allowing spin polarization (majority spin polarization positive and blue colored, minority spin polarization negative and red colored), (c) 2:1 cesium pentacene and, (d) 3:1 cesium pentacene. Fermi levels are indicated by black dashed lines.

\section{COMPUTATIONAL PROCEDURES}

Theoretical calculations for a number of new compounds able to show metallicity and, eventually, interesting superconducting properties have been performed using ab initio van der Waals-Density Functional Theory (vdW-DFT) as proposed in 2004 by Dion et al. $\stackrel{10}{ }$. Taking advantage of an algorithm introduced by Román-Peréz and Soler the whole calculation can be done in reciprocal space ${ }^{11}$. Actually, we have used a later development of the vdW-DFT approximation known as vdW-DFT2 ${ }^{12}$. It was coded by J. Klimeš within the VASP program ${ }^{13-16}$. Wavefunctions have been expanded in a planewave basis set up to a cutoff of $700 \mathrm{eV}$ and sampled on a $\Gamma$ centered Monkhorst-Pack grid automatically generated taking into account the sizes of the reciprocal lattice vectors (about 

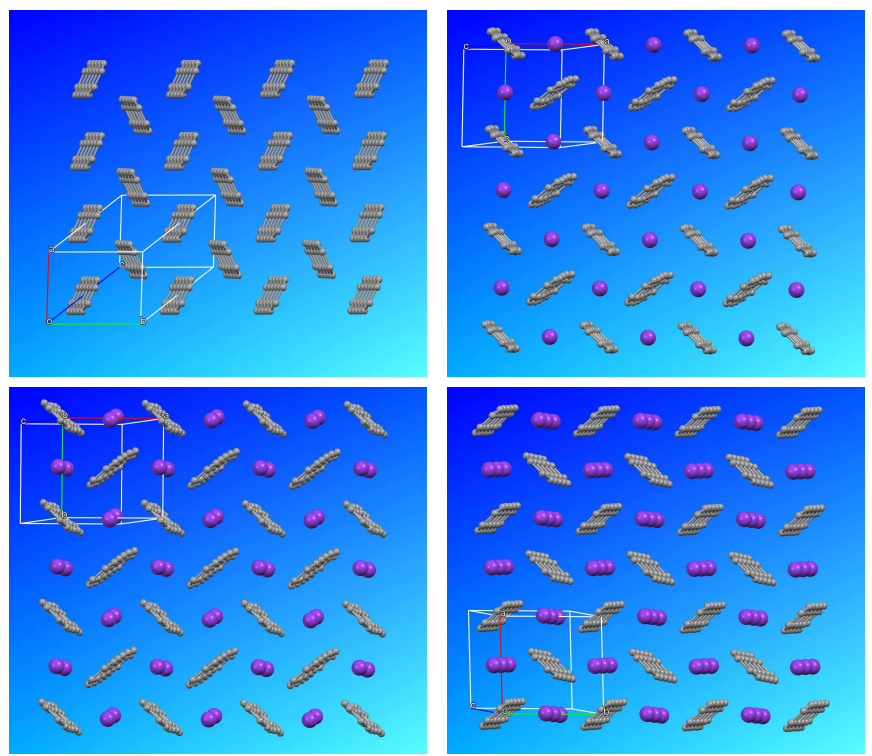

FIG. 4. (Color online) Rearrangement of pentacene molecules as cesium is intercalated into the herringbone crystalline structure of pristine pentacene. The addition of one Cs atom per pentacene suffices to change the angles between PAH molecules from $50.98^{\circ}$ to $86.97^{\circ}$. Further cesium addition enlarges the angles to $88.56^{\circ}$ and $88.72^{\circ}$ for 2:1 and 3:1 stoichiometries, respectively.

fifty points during geometry optimizations and two or three thousand to get precise densities of states using the tetrahedra method). Core electrons have been treated within the projector augmented method ${ }^{17,18}$. Semi-core $3 \mathrm{~s}$ and $3 p$ potassium states and $5 \mathrm{~s}$ and $5 \mathrm{p}$ cesium states have been considered valence states, i.e., they fully enter within the electronic density self-consistency loop. The relaxation of the electronic degrees of freedom has been stopped when both the total energy and the band structure energy variations between two steps are smaller than $10^{-8} \mathrm{eV}$. Ionic relaxation has been continued as long as any force were larger than $0.001 \mathrm{eV} / \AA^{19}$. The possibility of a breakdown of spin symmetry has always been considered. Results for spin-polarized bands are given in those cases in which polarization stabilizes the structure.

We have used free software GRACE maintained by Weizmann to get precise plots representing densities of states ${ }^{20}$ and a free version of MERCURY from The Cambridge Crystallographic Data Centre to visualize crystalline structures ${ }^{21}$. Structural data from VASP have been translated to Crystallographic Information File (cif) format using tools provided by Bilbao Crystallographic Server 22 .

\section{RESULTS AND DISCUSSION}

New possible structures for the compounds formed by intercalation of an alkali metal into a molecular crystal formed by polycyclic aromatic hydrocarbon (PAH) molecules have been theoretically searched for.

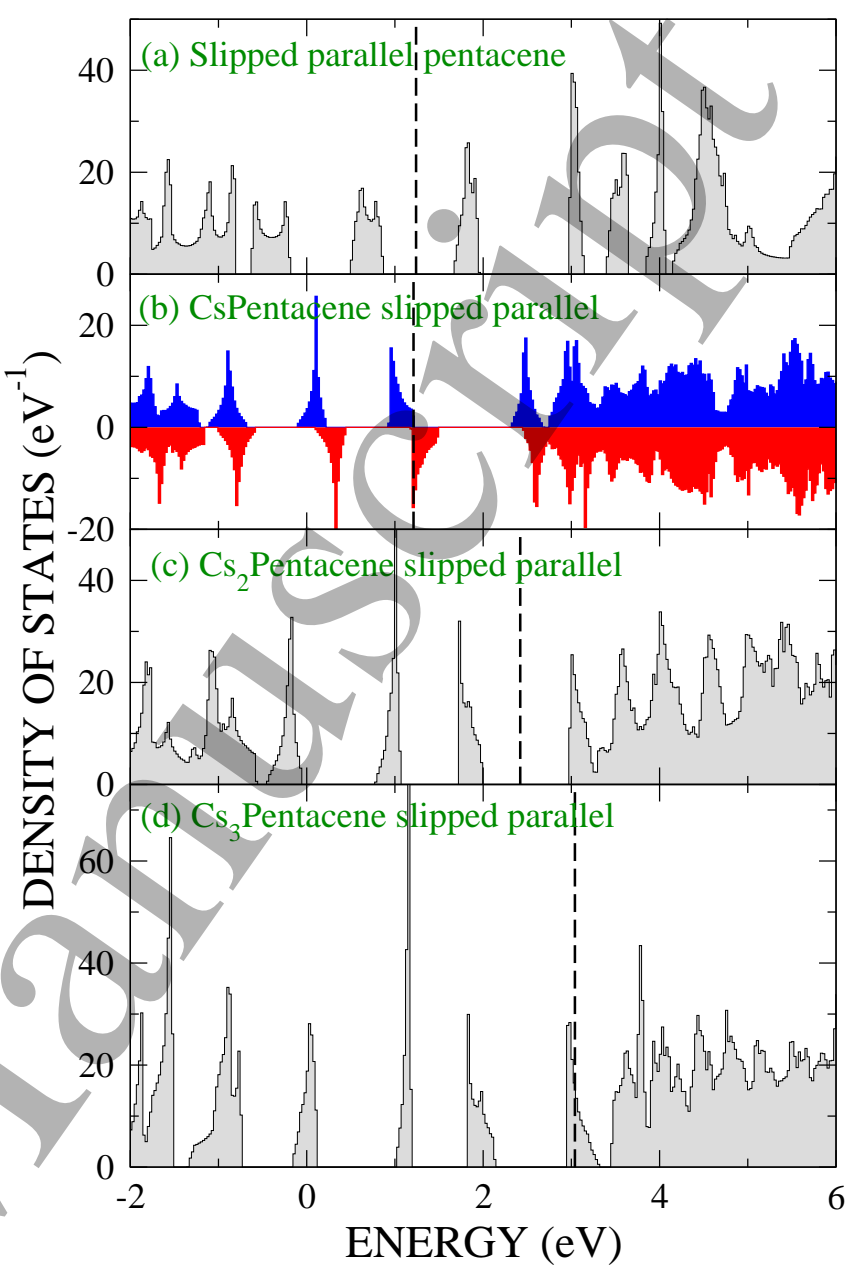

FIG. 5. (Color online) Density of states (DOS) changes due to intralayer cesium doping of pentacene in the slipped parallel structure. From top to bottom, (a) pristine pentacene, (in silico, non naturally occurring phase) (b) 1:1 cesium pentacene allowing spin polarization (majority spin polarization positive and blue colored, minority spin polarization negative and red colored), (c) 2:1 cesium pentacene and, (d) 3:1 cesium pentacene. Fermi levels are indicated by black dashed lines.

\section{A. Cesium Pentacene compounds from 1:1 to 3:1 stoichiometry}

An homogeneous compound formed by cesium and pentacene can be obtained by mechanical alloying following a recipe that is quite similar to the one used to synthesize potassium pentacene. As in that case, a maximum 3:1 CsPentacene stoichiometry can be reached ${ }^{23}$. Our computational study goes through the complete series, from pristine pentacene to $\mathrm{Cs}_{3}$ Pentacene. We start assuming the usual herringbone arrangement of pentacene molecules but later we switch to the alternative slipped parallel arrangement. Although the evolution of the density of states follows much the same patterns in both cases, energetic balances are quite different. 
TABLE I. Main properties of the studied compounds formed by the intercalation of an alkali metal (Cs or K) into a molecular crystal formed by polycyclic aromatic hydrocarbon (PAH) molecules (pentacene or picene). Crystals showing a herringbone arrangement of organic molecules imply primitive cells containing two molecules. On the other hand, while slipped parallel arrangement of picene molecules also favors primitive cells containing two molecules, pentacene slipped parallel arrangements allow smaller primitive cells containing just one organic molecule thanks to its axial symmetry. Volume, spin polarization and total energy are given per cell. Energies of formation are always given for pairs of organic molecules in order to make comparisons easier. Complete data of the corresponding crystalline structures are given in Supplemental Material as Crystallographic Information Files (cif files) (Ref. (38)).

\begin{tabular}{|c|c|c|c|c|c|}
\hline Compound & Structure (Space group) & Volume $\left(\AA^{3}\right)$ & Spin Polarization (e) & Total Energy $(\mathrm{eV})$ & Energy of Formation $(\mathrm{eV})$ \\
\hline \multirow[t]{2}{*}{ Potassium } & isolated atom & & 1 & 1.804 & $1 / 11$ \\
\hline & bcc crystal $(\operatorname{Im} \overline{3} \mathrm{~m})$ & 69.42 & 0 & 1.044 & -0.760 \\
\hline \multirow[t]{2}{*}{ Cesium } & isolated atom & & 1 & 1.838 & \\
\hline & bcc crystal (Im $\overline{3} \mathrm{~m})$ & 107.24 & 0 & 1.154 & -0.684 \\
\hline \multirow[t]{3}{*}{ Pentacene } & isolated molecule & & 0 & -216.071 & \\
\hline & herringbone crystal $(\mathrm{P} \overline{1})$ & 688.67 & 0 & -435.542 & -3.400 \\
\hline & slipped parallel crystal $(\mathrm{P} \overline{1})$ & 346.01 & 0 & -217.629 & -3.116 \\
\hline \multirow[t]{2}{*}{ Picene } & isolated molecule & & 0 & -216.703 & \\
\hline & herringbone crystal (P2 $\left.2_{1}\right)$ & 696.37 & 0 & -436.627 & -3.221 \\
\hline \multirow[t]{2}{*}{ CsPentacene } & herringbone crystal $(\mathrm{P} \overline{1})$ & 887.85 & 2 & -435.194 & -1.960 \\
\hline & slipped parallel crystal $(\mathrm{C} 2 / \mathrm{m})$ & 445.12 & 0.945 & -217.409 & -1.584 \\
\hline \multirow[t]{2}{*}{$\mathrm{Cs}_{2}$ Pentacene } & herringbone crystal $(\mathrm{P} \overline{1})$ & 910.16 & 0 & -436.146 & -5.220 \\
\hline & slipped parallel crystal $(\mathrm{P} \overline{1})$ & 456.05 & 0 & -217.865 & -4.804 \\
\hline \multirow[t]{3}{*}{$\mathrm{Cs}_{3}$ Pentacene } & herringbone crystal $(\mathrm{P} \overline{1})$ & 980.69 & 0 & -434.462 & -5.844 \\
\hline & slipped parallel crystal $(\mathrm{C} 2 / \mathrm{m})$ & 498.45 & 0 & -217.239 & -5.860 \\
\hline & slipped parallel crystal $(\mathrm{P} \overline{1})$ & 498.41 & 0 & -217.244 & -5.870 \\
\hline \multirow[t]{2}{*}{$\mathrm{K}_{3}$ Picene } & herringbone crystal $\left(\mathrm{P} 2_{1}\right)$ & 825.43 & 2 & -432.768 & -2.405 \\
\hline & slipped parallel crystal $\left(\mathrm{P} 2_{1} / \mathrm{m}\right)$ & 890.36 & 0 & -431.677 & -1.314 \\
\hline \multirow[t]{3}{*}{$\mathrm{Cs}_{3}$ Picene } & herringbone crystal $\left(\mathrm{P} 2_{1}\right)$ & 973.29 & 2 & -432.564 & -2.861 \\
\hline & slipped parallel crystal (P1) & 491.79 & 0 & -216.086 & -2.468 \\
\hline & slipped parallel crystal $(\mathrm{P} \overline{1})$ & 981.59 & 0 & -432.390 & -2.687 \\
\hline
\end{tabular}

Figure 3 shows the important band structure changes that follow an increasing alloying of pentacene with cesium. The first panel shows the density of states for pristine pentacene. A gap of $0.74 \mathrm{eV}$ separates unoccupied bands from occupied ones. The addition of an electron donor to crystalline pentacene immediately induce sensible changes in the relative orientation of organic molecules. Nearest-neighbor molecules move to an almost perpendicular configuration, that is, angles between molecule planes become approximately right. Figure 4 gives a graphical view of the organic molecules rearrangement. This kind of relaxation was previously observed for $\mathrm{K}_{x}$ Pentacene compounds $\%$. Figure 1 shows the structure at 3:1 stoichiometry. Following this structural change, band widths become smaller favoring metal-insulatớr transitions. Actually, LUMO bandwidth which is $0.725 \mathrm{eV}$ for pristine material (a) reduces to $0.2 \mathrm{eV}$ for CsPentacene before splitting in two spin-majority and spin-minority bands. Panel (b) shows our spin-polarized density of states with a tiny but real gap at the Fermi energy. Further addition of cesium leads to a structurally very robust insulator in which bands are completely occupied (or empty) and a $0.99 \mathrm{eV}$ gap separates conduction bands from valence bands (see panel (c)). Finally, at 3:1 stoichiometry, LUMO+1 orbital becomes half-occupied being spin-polarization unfavorable probably because the band is now a little wider, $0.35 \mathrm{eV}$ compared to $0.2 \mathrm{eV}$ for the $1: 1 \mathrm{Cs}$

\section{Slipped parallel arrangement of organic molecules}

Figure 5 shows the evolution of the density of states when cesium is added to a modified crystalline version of pristine pentacene based on a slipped parallel arrangement of the organic molecules. Although this modified phase of pure pentacene just exists in silico, its energy of formation lies only 0.3 $\mathrm{eV}$ per cell above the one obtained for the herringbone ordering which appears naturally (see below, Subsection IIIC). This structural framework is preserved after the addition of cesium from 1:1 to 3:1 stoichiometry. Panel (a) shows the density of states for pure pentacene in this stable although energetically unfavorable alternative structure. Bandwidths are significantly smaller than for the corresponding herringbone crystal. For example, LUMO bandwidth is here $0.3 \mathrm{eV}$ compared with corresponding herringbone value of $0.725 \mathrm{eV}$. Nevertheless, differences are much smaller after alloying. Evolution of band structure induced by increasing Cs addition follows the trends commented in previous subsection. 


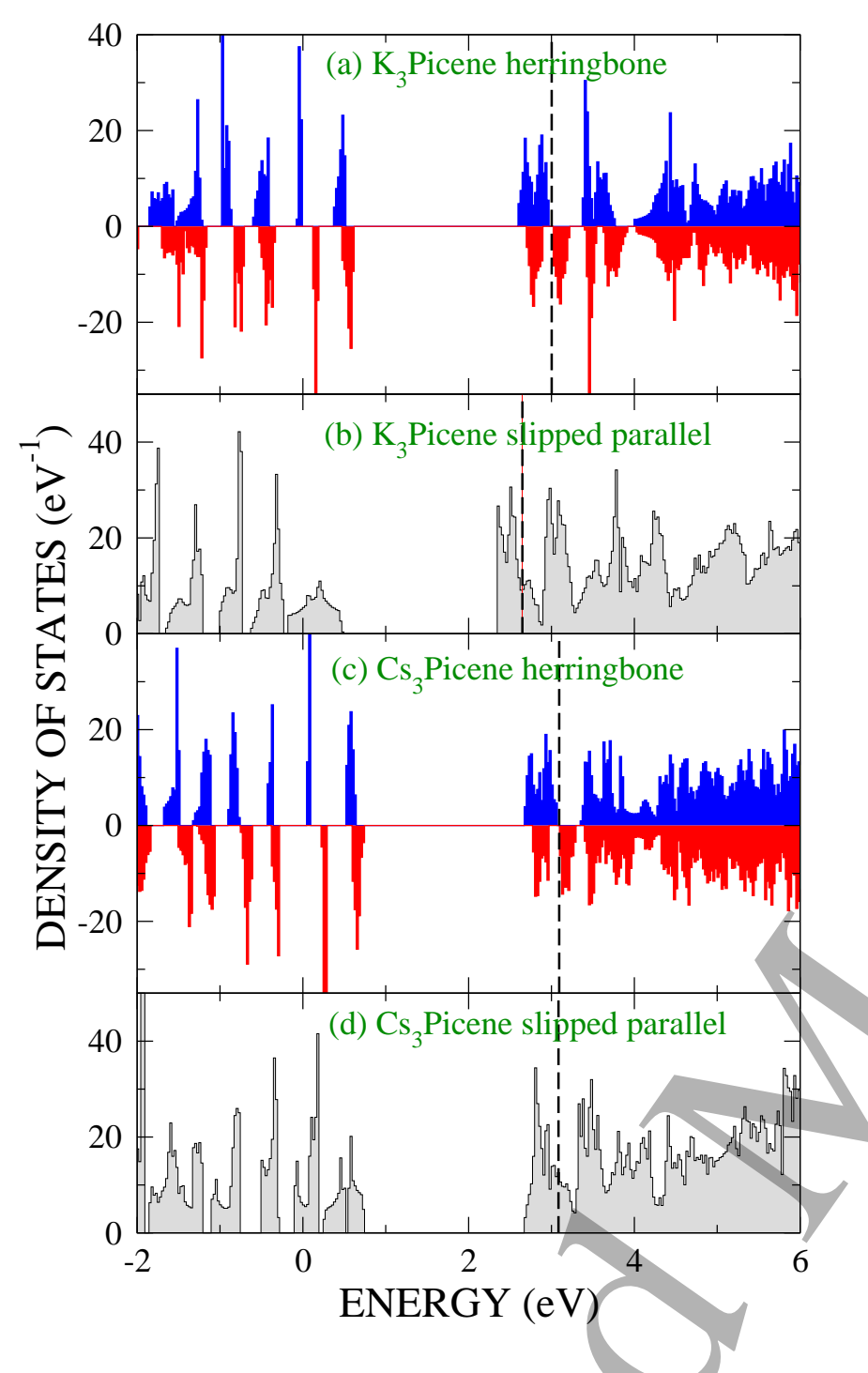

FIG. 6. (Color online) Changes in the density of states produced by potassium or cesium intercalation of picene. Both stable herringbone structure and the alternative slipped parallel arrangement have been considered. (a) 3:1 potassium picene allowing spin polarization (majority spin polarization positive and blue colored, minority spin polarization negative and red colored) in the herringbone structure, (b) 3:1 potassium picene in the alternative slipped parallel arrangement, (c) spin polarized 3:1 cesium picene in the herringbone structure and, (d) 3:1 cesium picene in a slipped parallel arrangement.

\section{B. Comparison with $X_{3}$ Picene compounds $(X=K, C s)$}

There are many papers dealing with the intercalation of picene with alkali or alkaline earth metals $-7,24-36$. None of them has considered the band structure of a slipped parallel arrangement of picene molecules. Even in the specific search looking for new structural patterns conducted by Naghavi and Tosatti using an evolutionary algorithm ${ }^{33}$, the prevalence of the herringbone arrangement was always patent. On the other hand, pentacene alloying has been systematically ignored un- til recently $\stackrel{8,9}{9}$. It is therefore very important to show the crucial differences that appear between picene and pentacene using a unified framework. Here we will show that picene alloying does not lead to a metallic behavior, either because the material experience a metal-insulator transition or because it is energetically non-viable ${ }^{37}$.

Figure 6 shows density of states for intercalated picene at its maximum stoichiometry, firstly with potassium and secondly with cesium. Both herringbone ((a)/and (c) panels) and slipped parallel ((b) and (d) panels) arrangements have been considered. Our results show that, from the band structure point of view alloying with potassium or cesium does make an important difference. In both cases, a metal-insulator transition for the herringbone structure occurs because bandwidths are small (unpolarized bandwidth is $0.2 \mathrm{eV}$ in both compounds). Actually, the existence of a metal-insulator transition for samples prepared with Cs was reported in the seminal work by Mitsuhashi et al. $\stackrel{1}{ }$. On the other hand, a metallic character is predicted for compounds showing a slipped parallel orientation of picene molecules both for $\mathrm{K}$ and $\mathrm{Cs}$ intercalation. This is due to the presence of wider bands and the merging of the band formed by LUMO+1 states into a continuous conduction band. Unfortunately, the syntheses of these compounds are expected to be challenging, at least as a mayor phase, given their unfavorable energy balance compared to their herringbone-structured counterparts. Energies of formation are particularly adverse for $\mathrm{K}_{3}$ Picene. Next subsection presents a complete analysis of the main properties of stable phases of all considered compounds with special emphasis on energies of formation.

\section{Energies of formation}

Table I compiles results for all the materials that have been considered in our study. Main properties are given for each compound, being of particular importance the vdW-DFT2 value of the total energy (per unit cell). From these data, energies of formation can be calculated. They constitute the cornerstone of our work. It should be pointed out at this point that the procedure we are using to predict crystalline phases was satisfactorily tested in our previous work on pentacene alloying 9 . We successfully reproduced there the experimental crystal structures of two naturally occurring polymorphs of pentacene, while regarding more specific interactions, the structure of the prototypical intercalation compound $\mathrm{KC}_{8}$ was correctly obtained as well. Energies of formation of the compounds studied in our work are obtained as differences between the calculated total energies of the respective stable phases of components. For example, the energy of formation per unit cell of $\mathrm{Cs}_{3}$ Pentacene from metallic cesium and pure pentacene is obtained by:

$$
\begin{gathered}
E\left(\mathrm{Cs}_{3} \text { Pentacene }\right)-E(\text { Pentacene })-6 E(\mathrm{Cs})= \\
(-434.462-(-435.542)-6 \times 1.154) \mathrm{eV}=-5.844 \mathrm{eV},
\end{gathered}
$$


using data corresponding to herringbone structures or:

$$
\begin{gathered}
2 E\left(\mathrm{Cs}_{3} \text { Pentacene }\right)-E(\text { Pentacene })-6 E(\mathrm{Cs})= \\
(2 \times(-217.244)-(-435.542)-6 \times 1.154) \mathrm{eV}=-5.870 \mathrm{eV},
\end{gathered}
$$

using data corresponding to the slipped parallel structure for the compound but herringbone structural data for pristine pentacene and taking into account that slipped parallel cell contains half the ingredients of the herringbone cell.

Data in Table I show that stoichiometry going from 1:1 to 3:1 are possible for Cs-Pentacene alloying since energies of formation are ever decreasing and systematically negative. It is also clear that 2:1 manifests a stronger relative stabilization energy that is in line with recent experimental findings $s^{6.7}$. Disproportionation of a $\mathrm{Cs}_{3}$ Pentacene compound into $\mathrm{Cs}_{2}$ Pentacene plus cesium is therefore energetically unfavorable by $0.62 \mathrm{eV}$ per unit cell using total energies values from Table I:

$$
\begin{gathered}
E\left(\mathrm{Cs}_{2} \text { Pentacene }\right)+2 E(\mathrm{Cs})-E\left(\mathrm{Cs}_{3} \text { Pentacene }\right)= \\
(-436.146+2 \times 1.154-(-434.462)) \mathrm{eV}=0.624 \mathrm{eV} .
\end{gathered}
$$

Data in Table I show two trends that we would like to emphasize. Firstly, formation energies of cesium pentacene compounds are considerable larger than that of metal-picene alloys. Analogous values for potassium pentacene compounds have been also obtained 9 . Therefore, the specific PAH molecule coming into the compound matters much more than the selected metal. Secondly, although $\mathrm{Cs}_{3}$ Picene energies of formation are similar for the two structural phases that we are analyzing, only $\mathrm{Cs}_{3}$ Pentacene prefers the formation of a slipped parallel crystal. The consequences of this fact are important since the above reported band structure data show that metallicity is mainly obtained for this molecular arrangement. Remember the DOS results presented in Fig.6showing metal-

* jav@icmm.csic.es

1 R. Mitsuhashi, Y. Suzuki, Y. Yamanari, H. Mitamura, T. Kambe, N. Ikeda, H. Okamoto, A. Fujiwara, M. Yamaji, N. Kawasaki, Y. Maniwa, and Y. Kubozono, Nature 464, 76 (2010).

2 Y. Kubozono, R. Eguchi, H. Goto, S. Hamao, T. Kambe, T. Terao, S. Nishiyama, L. Zheng, X. Miao, and H. Okamoto, J. Phys.: Condens. Matter 28, 334001 (2016).

3 G.A. Artioli and L. Malavasi, J. of Mater. Chem. C 2, 1577 (2014).

${ }^{4}$ S. Heguri, M. Kobayashi, and K. Tanigáki, Phys. Rev. B. 92, 014502 (2015).

${ }^{5}$ See, for example, A. Guijarro, J.A. Vergés, E. San-Fabián, G. Chiappe, and E. Louis, Chem. Phys. Chem. 17, 3548 (2016), for a general discussion of some PAH families.

${ }^{6}$ Y. Takabayashi, M. Menelaou, H. Tamura, N. Takemori, T. Koretsune, A. Štefančič, G. Klupp, A.J.C. Buurma, Y. Nomura, R. Arita, D. Aron, M.J. Rosseinsky, and K. Prassides, Nature Chem. 9, 635 (2017).

${ }^{7}$ F.D. Romero, M.J. Pitcher, C.I. Hiley, G.F.S. Whitehead, S. Kar, A.Y. Ganin, D. Antypov, C. Collins, M.S. Dyer, G. Klupp, R.H. Colman, K. Prassides, and M.J. Rosseinsky, Nature Chem. 9, 644 (2017). insulator transitions for $\mathrm{X}_{3}$ Picene $(\mathrm{X}=\mathrm{K}, \mathrm{Cs})$ in the more stable herringbone structures.

\section{CONCLUDING REMARKS}

Our numerical study of plausible crystalline structures for potassium or cesium intercalated pentacene or picene hydrocarbons provides a general conclusion. Both types of crystal lattices (intercalated derivatives of pentacene vs. picene) have important overall structural similarities, and display as a result similar calculated band structures. These observed regularities should induce a shift in the main strategies in the research of intercalated PAH superconductivity towards the exploration of new types of intermolecular packing architectures, moving the often invoked issue of the electronic configuration of the individual PAHs to a secondary role. Among the currently explored structures in this work, $\mathrm{Cs}_{3}$ Pentacene seems to depart from a privileged position since the two proposed molecular arrangements are energetically feasible and point towards half-filled metallic bands. In any case, potassium alloying is not the best choice because compounds tend to preserve the herringbone frameworks of pure picene and pentacene resulting in very narrow bands that undergo metal-insulator transitions.

\section{ACKNOWLEDGMENTS}

Financial support by the Spanish Ministry of Economy and Competitiveness (FIS2015-64222-C2-1-P and MAT201678625-C2-2-P), the Generalitat Valenciana (Grant PROMETEO/2017/139) and the University of Alicante is gratefully acknowledged.

${ }^{8}$ T. Nakagawa, Z. Yuan, J. Zhang, K.V. Yusenko, C. Drathen, Q. Liu, S. Margadonna, and C. Jin, J. Phys.: Condens. Matter 28, 484001 (2016).

9 A. Guijarro and J.A. Vergés, Phys. Rev. B. 95, 134112 (2017).

10 M. Dion, H. Rydberg, E. Schröder, D.C. Langreth, and B.I. Lundqvist, Phys. Rev. Lett. 92, 246401 (2004).

11 G. Román-Pérez and J.M. Soler, Phys. Rev. Lett. 103, 096102 (2009).

12 K. Lee, E. D. Murray, L. Kong, B.I. Lundqvist, and D.C. Langreth, Phys. Rev. B. 82, 081101 (2010).

13 J. Klimeš, D.R. Bowler, and A. Michaelides, Phys. Rev. B. 83, 195131 (2011).

${ }^{14}$ G. Kresse and J. Hafner, Phys. Rev. B. 47, 558 (1993); ibid 49, 14251 (1994).

15 G. Kresse and J. Furthmüller, Comput. Mat. Sci. 6, 15 (1996).

${ }^{16}$ G. Kresse and J. Furthmüller, Phys. Rev. B. 54, 11169 (1996).

17 P.E. Blöchl, Phys. Rev. B. 50, 17953 (1994).

18 G. Kresse and D. Joubert, Phys. Rev. B. 59, 1758 (1999).

19 This set of parameters together with the tag accurate within the VASP input seems to work correctly even for compounds formed by a mixture of organic molecules and alkaline elements. Actu- 
ally, the selected energy cutoff of $700 \mathrm{eV}$ is well above the recommended values for C, H, Cs and K PAW potentials which are 400, 250,220 and $259 \mathrm{eV}$, respectively. Two realistic tests discussed in Ref. 9, polymorphism in pristine pentacene and the structure of the $\mathrm{KC}_{8}$ intercalation compound, were shown to give satisfactory result using the same numerical procedure. See also the discussion at the beginning of subsection IIIC.

${ }^{20}$ Grace is a What You See Is What You Get 2D plotting tool for the $\mathrm{X}$ Window System and $\mathrm{M} *$ tif. Software at http://plasma-gate.weizmann.ac.il/Grace.

21 Mercury: Crystal Structure Visualisation, Exploration and Analysis Made Easy. Cambridge Crystallographic Data Centre. See, http: //www.ccdc.cam.ac.uk/mercury/.

22 See Structure Data Converter \& Editor at Bilbao Crystallographic Server (http: / / www. cryst.ehu.es).

${ }^{23}$ Synthesis of Cs pentacene compounds has been recently achieved by Guijarro's organic chemistry group in Alicante (to be published). Computational simulation shows that metallic content exceeding three atoms per PAH molecule goes to interlayer planes instead of remaining between organic molecules.

${ }^{24}$ P. L. de Andres, A. Guijarro, and J. A. Vergés, Phys. Rev. B. 83, 245113 (2011)

${ }^{25}$ M. Kim, B.I. Min, G. Lee, H.J. Kwon, Y.M. Rhee, and J.H. Shim, Phys. Rev. B. 83, 214510 (2011).

${ }^{26}$ T. Kosugi, T. Miyake, S. Ishibashi, R. Arita, and H. Aoki, Phys. Rev. B. 84, 214506 (2011).

27 G. Giovannetti and M. Capone, Phys. Rev. B. 83, 134508 (2011).

28 M. Casula, M. Calandra, G. Profeta, and F. Mauri, Phys. Rev. Lett. 107, 137006 (2011).

29 A. Ruff, M. Sing, R. Claessen, H. Lee, M. Tomić, H.O. Jeschke, and R. Valentí, Phys. Rev. Lett. 110, 216403 (2013).

30 T. Kambe, X. He, Y. Takahashi, Y. Yamanari, K. Teranishi, H. Mitamura, S. Shibasaki, K. Tomita, R. Eguchi, H. Goto, Y. Tak- abayashi, T. Kato, A. Fujiwara, T. Kariyado, H. Aoki, and Y. Kubozono, Phys. Rev. B. 86, 214507 (2012).

${ }^{31}$ K. Teranishi, X. He, Y. Sakai, M. Izumi, H. Goto, R. Eguchi, Y. Takabayashi, T. Kambe, and Y. Kubozono, Phys. Rev. B. 87, 060505(R) (2013).

${ }^{32}$ G-H. Zhong, C. Zhang, G-F. Wu, Z-B. Huang, X-J. Chen, and H-Q. Lin, J. of Applied Phys. 113, 17E131 (2013).

33 S.S. Naghavi and E. Tosatti, Phys. Rev. B. 90, 075143 (2014).

${ }^{34}$ G-H. Zhong, C. Zhang, H-Q. Lin, IEEE Trans. on Magnetism 50, 1700103 (2014).

35 S. Heguri, M. Kobayashi, and K. Tanigaki, Phys. Rev. B. 92, 014502 (2015).

${ }^{36}$ T. Kambe, S. Nishiyama, H.L.T. Nguyen, T. Terao, M. Izumi, Y. Sakai, L. Zheng, H. Goto, Y. Itoh, T. Onji, T.C. Kobayashi, H. Sugino, S. Gohda, H. Okamoto and Y. Kubozono, J. Phys.: Condens. Matter 28, 444001 (2016).

37 Reference 1 showed superconductivity at two different critical temperatures for $\mathrm{K}_{x}$ Picene compounds with $2.6 \leq x \leq 3.3$. The shielding fraction of the material was very small for all studied samples. Our theoretical results are only compatible with these experimental findings if the energetically disfavored slipped parallel phase of $\mathrm{K}_{3}$ Picene is just the shielding fraction producing the diamagnetic signal. Actually, superconducting samples prepared with rubidium show larger shielding fractions, a result that is compatible with the smaller energy differences that we find for cesium compounds. (We do not have results for $\mathrm{Rb}$ compounds but logically they will interpolated between $\mathrm{K}$ and Cs results).

See Supplemental Material at http:www $x x \cdot y y \cdot z z$ for a collection of all relevant crystalline structures mentioned in the main text. They are given in Crystallographic Information File (cif) format as defined by International Union of Crystallography. We have systematically used the Structure Data Converter \& Editor at Bilbao Crystallographic Server (Ref.(22)) to transform VASP structural data to cif format and vice versa. 